\title{
Modelado y análisis formal de jugadas del fútbol
}

\author{
Jonathan Tellez-Giron, Matías Alvarado \\ Centro de Investigación y de Estudios Avanzados del IPN, CDMX, \\ México
}

\begin{abstract}
Resumen. Desarrollamos un modelado analítico del fútbol soccer, y se proponen fórmulas para medir la ocurrencia promedio de jugadas por minuto y la eficiencia de un jugador en cada posición de juego. Con datos de la Liga Española sobre la ocurrencia de jugadas por jugador, se validan las formulas propuestas. La secuencia correcta de jugadas se logra mediante la función de transición de un autómata finito para el futbol.
\end{abstract}

Palabras clave: Modelo de fútbol soccer, autómatas concurrentes, eficiencia de jugador.

\section{Modeling and Formal Analysis of Football Plays}

\begin{abstract}
We present an analytical modeling of soccer football, and formulas to measure the average occurrence of plays per minute and the efficiency of a player in each playing position are proposed . Using data from the Spanish League on the occurrence of plays per player, we validate the proposed formulas. The correct sequence of moves is by means of the transition function of a finite automaton to soccer football.
\end{abstract}

Keywords: Soccer football modeling, concurrent finite automaton, player's efficiency.

\section{Introducción}

El fútbol soccer (FS), del ingles football o balompié, es uno de los juegos más populares a nivel mundial, inventado en la gran Bretaña desde la Edad Media y trasladado fuera por los marineros británicos. A mediados del siglo XVII estudiantes de la universidad de Cambridge desarrollaron las primeras reglas oficiales para este deporte $[3$. El fútbol se juega en una cancha de césped de $120 \times 60 \mathrm{~m}$, con arcos o porterías en cada lado del campo, entre dos equipos de 11 jugadores cada uno. Un partido de fútbol inicia con el balón a media cancha; un jugador del equipo que ganó el volado para dar el primer toque al balón lo pasa a un compañero. Sobre el césped los jugadores deben conducir el balón con los pies y pueden controlarlo, asimismo, con piernas, cuerpo y cabeza, pero no con brazos ni manos, salvo para los saques de banda. A base de conducción 
individual y pases del balón entre compañeros y de esquivar a los rivales, deben buscar introducir el balón en la portería rival para anotar goles; gana el partido el equipo que anota mayor número de goles. Los roles de juego, acorde a su posición en la cancha respecto a la portería propia son delanteros, medios, defensas, y el portero, siendo la misión del portero evitar anotaciones de gol en su portería, y sólo él tiene permitido el manejo del balón con manos y brazos.

En el FS, como en todo juego, los jugadores pretende realizar la mejor acción posible en beneficio del equipo, con base en elegir la mejor estrategia considerando las condiciones del juego y, especialmente, las estrategias del resto de los jugadores. La selección de estrategias es un aspecto esencial a considerar en la automatización del FS. Actualmente, el modelado formal y el análisis estratégico para juegos multi-jugador como el fútbol americano o el béisbol [241], es relevante en la ciencia de los deportes, la computación y la teoría de juegos.

A continuación se presentan las jugadas del FS, haciendo mención de las jugadas de cada jugador, clasificadas de acuerdo a su posición, en la Tabla 1 . delanteros, mediocampistas, defensas y portero. El conjunto de jugadas en la Tabla 1, se tomaron de la página web oficial Liga BBVA y la (http://mex. laliga.es/liga-bbva), donde se muestra los datos de cada equipo, los datos relevantes por jugador, así como el conteo de jugadas relevantes. La página web se dividen en cinco grandes categorías: jugadas defensivas, jugadas de construcción, jugadas de ataque, jugadas de gol y por último una categoría que refleja la disciplina de los jugadores.

\section{Ocurrencia de jugadas y eficiencia de jugadores}

\subsection{Frecuencia de jugadas}

Las jugadas para las posiciones de delantero, mediocampista y defensa son las mismas, porque para ellos no existen reglas que limiten su movilidad en el campo. La diferencia por rol es la probabilidad de que suceda una jugada especifica: por ejemplo, un delantero puede hacer una barrida para quitar el balón sin embargo la probabilidad de que un delantero promedio haga una recuperación (por minuto) es de $4.62 \%$ mientras que de un defensa es de $6.44 \%$; inversamente, podemos observar que la probabilidad de que un defensa anote gol es de $0.05 \%$ mientras que la probabilidad de que un delantero anote gol es de $0.99 \%$. El portero tiene jugadas propias, dada su función de evitar que el equipo contrario anote gol y su posibilidad de utilizar las manos para lograr su objetivo.

Para el análisis se toman en cuenta el número de ocurrencias de las jugadas para los jugadores mas populares de cada equipo, así como a los equipos mejor clasificados conforme los puntos obtenidos durante el torneo; asimismo, la diferencia entre goles anotados y recibidos. En caso de empate de puntos entre tres o más equipos se considera la misma diferencia en los partidos que involucren a dichos equipos, y los goles anotados en total a lo largo de la liga. Los datos son de la primera jornada en agosto 2015 hasta la jornada 30 del torneo en marzo 2016. 
Tabla 1. Clasificación de Jugadas en el FS.

Jugadas defensivas

\begin{tabular}{cl|cl|cl} 
Jugada & Descripción & Jugada & Descripción & Jugada & Descripción \\
\hline$d b^{i}$ & bloquear disparo & $r^{i}$ & recuperación & $i^{i}$ & intercepción \\
$d^{i}$ & despejar & blce & bloqueo con éxito & blse & bloqueo sin éxito
\end{tabular}

\section{Jugadas de construcción}

\begin{tabular}{c|c|c} 
Jugada Descripción & Jugada Descripción & Jugada Descripción \\
\hline$p l^{j} \quad$ pase largo & $p c^{i}$ pase corto & $c e^{i}$ centro
\end{tabular}

Jugadas de ataque

\begin{tabular}{|c|c|c|}
\hline Jugada Descripción & Jugada Descripción & Jugada Descripción \\
\hline $\begin{array}{cl}t^{i} & \text { tiro } \\
r e f^{i} & \text { regate fallido }\end{array}$ & $a s^{i} \quad$ asistencia & $r e a^{i}$ regate acertado \\
\hline
\end{tabular}

Jugada de gol

\begin{tabular}{cc} 
Jugada & Descripción \\
\hline go $^{i}$ & gol
\end{tabular}

\section{Disciplina}

\begin{tabular}{cl|cl|cl} 
Jugada & Descripción & Jugada & Descripción & Jugada & Descripción \\
\hline$f a p^{i}$ & falta propia & $r e p^{i}$ & recibir penalti & $f u j^{i}$ & fuera de juego \\
$f a r^{i}$ & falta recibida & $p e c^{i}$ & cometer penalti & $p e r^{i}$ & perder balón \\
$m a c^{i}$ & cometer mano & $a d v^{i}$ & advertencia & $e x p^{i}$ & expulsión \\
$s d u^{i}$ & sanción débil uno & $s d d^{i}$ & sanción débil dos & $s f^{i}$ & sanción fuerte \\
$s a^{i}$ & sanción acumulada & & & &
\end{tabular}

\section{Jugadas de Portero}

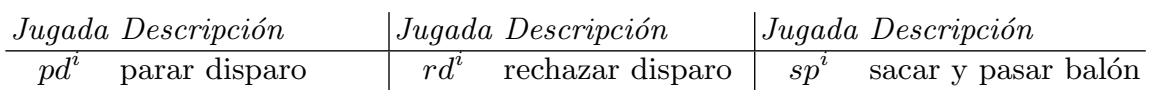

Con base en dichos datos se hace un análisis estadístico para obtener la ocurrencia de las jugadas por jugador. El tiempo de juego por jugador es variable debido a posibles cambios y/o expulsiones a lo largo de los encuentros. Así, la cantidad de minutos jugados puede variar incluso teniendo la misma cantidad de partidos jugados. Estos datos se normalizaron obteniendo la cantidad promedio de jugadas por partido y por minutos jugados para cada uno de los jugadores. Esto permite obtener una ocurrencia promedio por minuto jugado, cuyos valores se muestran en la Tabla 2 .

\subsection{Eficiencia de un jugador}

A partir de la ocurrencia total de jugadas en la Liga calculamos: 
- La ocurrencia promedio de jugadas por partido, ecuación 1.

- La ocurrencia promedio de jugadas por minuto, ecuación 2

$$
\begin{array}{r}
O P P=\frac{\sum_{i=1}^{N} \frac{j(i)}{p(i)}}{N}, \\
O P M=\frac{\sum_{i=1}^{N} \frac{j(i)}{m(i)}}{N},
\end{array}
$$

donde, $O P P=$ ocurrencia promedio de jugadas por partido, $O P M=$ ocurrencia promedio de jugadas por minuto, $N=$ número de jugadores, $j(i)=$ número de jugadas en la Liga por el jugador $i, p(i)=$ número de partidos jugados por el jugador $i, m(i)=$ número de minutos jugados por el jugador $i$. Estos datos se utilizan para calcular la eficiencia de cada jugador. De forma analítica, la $O P M$ permite mayor precisión y evita ambigüedad en cuanto a la eficiencia de un jugador. Por ejemplo, si un jugador esta presente pocos minutos de un partido, y en pocos minutos es capaz de realizar una anotación, entonces dicho jugador es muy eficiente. Por tanto nuestro interés se centra en la eficiencia por minutos jugados (emj).

Tabla 2. Ocurrencia promedio por minutos jugados para delanteros en Liga BBVA 2015/2016.

\begin{tabular}{ccc|ccc} 
Jugada & Ocurrencia & Probabilidad & Jugada & Ocurrencia & Probabilidad \\
\hline$d^{i}$ & 0.0068 & 0.00013104 & rep $^{i}$ & 0.0000 & 0.00000000 \\
$p l^{j}$ & 0.0168 & 0.02439200 & $r e a^{i}$ & 0.0106 & 0.00020360 \\
c $^{i}$ & 0.3349 & 0.48752592 & go $^{i}$ & 0.0068 & 0.00991233 \\
$c e^{i}$ & 0.0130 & 0.01893542 & fap $^{i}$ & 0.0151 & 0.02203089 \\
$t^{i}$ & 0.0296 & 0.04312250 & far $^{i}$ & 0.0184 & 0.02680279 \\
$a s^{i}$ & 0.0028 & 0.00005410 & $a d v^{i}$ & 0.0130 & 0.01888120 \\
$r e f^{i}$ & 0.0130 & 0.00025002 & sdu $^{i}$ & 0.0021 & 0.00308974 \\
$r^{i}$ & 0.0317 & 0.00060920 & sdd $^{i}$ & 0.0000 & 0.00005995 \\
$i^{i}$ & 0.0000 & 0.00000000 & $s f^{i}$ & 0.0000 & 0.00000000 \\
$f u j^{i}$ & 0.0099 & 0.01446414 & blce $^{i}$ & 0.0074 & 0.00014196 \\
$b l s e^{i}$ & 0.0028 & 0.00005383 & per $^{i}$ & Determinado \\
$p e c^{i}$ & 0.0000 & 0.00005994 & sa $^{i}$ & por otras \\
mac $^{i}$ & 0.0011 & 0.00165763 & exp $^{i}$ & jugadas \\
$d b^{i}$ & 0.0055 & 0.00010564 & \multicolumn{4}{|c}{} \\
\hline
\end{tabular}

El análisis de emj para los jugadores tuvo el objetivo de diferenciar las características principales de los diferentes roles y su desempeño en cada una de ellas. Los criterios de evaluación de emj son : bloqueos, duelos cuerpo a cuerpo, duelos aéreos, pases largos, pases cortos, centros, regates, penaltis y goles. La emj de los criterios mencionados se calcula a partir de la siguiente formula: 


$$
\mathbf{e m j}=\frac{\text { Ocurrencia promedio de jugadas exitosas por minutosjugados }}{\text { Ocurrencia promedio de jugadas totales por minutosjugados }}
$$

Por ejemplo, en el caso de los goles se calcula como

$$
\frac{\text { Goles promedio por minutosjugados }}{\text { Cantidad de tiros promedio por minutosjugados }} \text {. }
$$

Con este análisis se obtuvieron resultados tales que permiten determinar, claramente, la emj de cada rol. Los resultados se muestran en la Tabla 3. El comparativo de eficiencia entre jugadores de la Liga BBVA en la Tabla 4 La emj de un delantero promedio es un indicador de contraste respecto a la emj del resto de los jugadores: la eficiencia de Lionel Messi en pases cortos (82.6\%) sobre el promedio $(73.39 \%$ ) identifica a este jugador como coordinador de juego del equipo; a diferencia, el porcentaje de Luis Suarez en goles es $27.08 \%$ sobre el promedio (22.53\%), indicando que su rol en el juego es de caza-goles. De este análisis puede verse cómo, a pesar de que ambos jugadores pertenecen al mismo equipo y tienen el mismo rol como delanteros, su función individual se caracteriza acorde a su eficiencia en determinados aspectos del juego. La habilidad del jugador es factor primordial durante el desarrollo de un juego, caracteriza su rol en el campo e indica una preferencia sobre sus elecciones estratégicas.

Tabla 3. Eficiencia promedio por minutos jugados (\%) en la Liga BBVA 2015/2016.

\begin{tabular}{|c|c|c|c|c|c|c|c|c|c|c|}
\hline $\begin{array}{c}\text { Jugador } \\
\text { Promedio }\end{array}$ & Bloqueo & $\begin{array}{c}\text { Duelos } \\
\text { cuerpo } \\
\text { a } \\
\text { cuerpo }\end{array}$ & $\begin{array}{c}\text { Duelos } \\
\text { aéreos }\end{array}$ & $\begin{array}{c}\text { Pases } \\
\text { largos }\end{array}$ & $\begin{array}{c}\text { Pases } \\
\text { cortos }\end{array}$ & Centros & $\begin{array}{l}\text { Tiros a } \\
\text { puerta }\end{array}$ & Regates & Penaltis & Goles \\
\hline $\begin{array}{c}\text { Delantero } \\
\text { Medio }\end{array}$ & 72.62 & 42.85 & 38.39 & 57.06 & 73.39 & 19.58 & 55.25 & 45.49 & 23.75 & 22.53 \\
Defensa & 71.28 & 52.60 & 45.23 & 62.55 & 83.13 & 25.99 & 40.58 & 56.97 & 5.26 & 8.38 \\
\end{tabular}

Tabla 4. Eficiencia de jugadores por minutos jugados (\%) en la Liga BBVA 2015/2016.

\begin{tabular}{|c|c|c|c|c|c|c|c|c|c|c|}
\hline Jugador & Bloqueos & $\begin{array}{c}\text { Duelos } \\
\text { cuerpo } \\
\text { a } \\
\text { cuerpo }\end{array}$ & $\begin{array}{c}\text { Duelos } \\
\text { aéreos }\end{array}$ & $\begin{array}{c}\text { Pases } \\
\text { largos }\end{array}$ & $\begin{array}{c}\text { Pases } \\
\text { cortos }\end{array}$ & Centros & $\begin{array}{c}\text { Tiros a } \\
\text { puerta }\end{array}$ & Regates & Penaltis & Goles \\
\hline Luis Suárez & 82.35 & 43.4 & 46.43 & 48.48 & 72.95 & 6.9 & 55.21 & 35.56 & 33.33 & 27.08 \\
Lionel Messi & 62.5 & 54.95 & 25 & 72.41 & 82.6 & 21.43 & 63.33 & 65.33 & 42.86 & 24.44 \\
Neymar & 61.9 & 54.86 & 43.33 & 74.63 & 81.52 & 30.77 & 56.04 & 58.08 & 66.67 & 23.08 \\
Griezmann & 76 & 47.01 & 33.1 & 78.18 & 79.01 & 36.84 & 55.93 & 48.48 & 50 & 28.81 \\
Fernando Torres & 75 & 38.93 & 49.5 & 25 & 67.52 & 40 & 47.83 & 42.11 & 0 & 21.74 \\
Cristiano Ronaldo & 80 & 46.24 & 56.04 & 60 & 80.04 & 16.28 & 52.5 & 52.05 & 66.67 & 17.5 \\
Karim Benzema & 75 & 41.84 & 28.57 & 85.71 & 81.93 & 5.56 & 64.62 & 45.71 & 0 & 30.77 \\
Gareth Bale & 75 & 53.7 & 30 & 73.33 & 80.24 & 23.53 & 50 & 58.18 & 0 & 27.78 \\
Soldado Rillo & 55 & 49.71 & 24.68 & 52.17 & 69.22 & 9.52 & 54.55 & 55 & 0 & 12.12 \\
Cedric Bakambu & 80 & 34.59 & 25.53 & 63.64 & 73.5 & 15 & 65 & 58.62 & 0 & 27.5 \\
\hline
\end{tabular}


En resumen, el análisis de los datos estadísticos o de frecuencia de ocurrencia de jugadas del FS, expresa de manera realista, lo que cada jugador puede realizar según las condiciones de ocurrencia de las jugadas.

\section{Lenguaje formal y autómata finito para el fútbol}

El estudio previo sobre la frecuencia de ocurrencia de jugadas, permite obtener fotografías de la eficiencia de los jugadores en cierto momento de un torneo. Para modelar en general la dinámica del FS y las combinaciones que pueden ocurrir, es necesario otra herramienta de carácter algorítmico, tal que permita la simulación dinámica de cualquier partido posible. Se construyó una Gramática Libre de Contexto y un Autómata Finito (AF) para tal fin. Los estados principales son: cuando un jugador está en posesión y sin posesión del balón. A partir de estos estados se modela el FS para todo jugador, con excepción del portero. El conjunto de estados del jugador se presenta en la Tabla 5.

Tabla 5. Conjunto de estados de un jugador en el fútbol.

\begin{tabular}{cl|cl} 
Estado Descripción & Estado Descripción \\
\hline$P$ & Posesión del balón & $X$ & Estado posterior a expulsión \\
$S P$ & Sin posesión del balón & $F J$ & Estado posterior a fuera de juego \\
$G$ & Estado posterior a un gol & $T A$ & Tarjeta amarilla \\
$F$ & Estado posterior a una falta & $S T A$ & Segunda tarjeta amarilla \\
$T R$ & Tarjeta Roja & & \\
\hline
\end{tabular}

Las transiciones entre los estados de juego son a través de las jugadas posibles en cada estado. La función de transición del AF se define como $\delta: Q \times \Sigma \rightarrow Q$, donde $Q$ es el conjunto finito de estados y $\Sigma$ es el alfabeto, en este caso de las jugadas de FS. Así, la función define las transiciones de un estado hacia otro, a través de las jugadas que se dan en la Tabla 6.

\subsection{Construcción de jugadas}

El AF, ilustrado en la Figura 1, es definido como sigue:

- $Q=\{P, S P, G, F, T R, X, F J, T A, S T A\}$,

- $\Sigma=\left\{d^{i}, p l^{j}, p c^{i}, c e^{i}, t^{i}, a s^{i}, r e f^{i}, r^{i}, i^{i}, e x p^{i}, r e p^{i}, r e a^{i}, g o^{i}, f a p^{i}, f a r^{i}, a d v^{i}\right.$, $\left.s d u^{i}, s d d^{i}, s f^{i}, s a^{i}, f u j^{i}, p e r^{i}, d b^{i}, p e c^{i}, m a c^{i}, s p^{i}, p d^{i}, r d^{i}, b l c e^{i}, b l s e^{i}\right\}$,

- $\delta$ : función de transición, definida en Tabla 6 .

- $q_{0}=P$,

- $F=\{G, X\}$.

Cada transición del AF corresponde a una regla de la GLC -la cual hemos omitido. Se diseño un algoritmo con base en el AF definido previamente, que se 
Tabla 6. Transiciones entre estados de un jugador en el fútbol.

\begin{tabular}{lc|lc|lc} 
Función & Transición & Función & Transición & Función & Transición \\
\hline$\delta\left(P, f a r^{i}\right)$ & $\mathrm{P}$ & $\delta\left(P, t^{i}\right)$ & $\mathrm{SP}$ & $\delta\left(S P, i^{i}\right)$ & $\mathrm{P}$ \\
$\delta\left(P, r e p^{i}\right)$ & $\mathrm{P}$ & $\delta\left(P, a s^{i}\right)$ & $\mathrm{SP}$ & $\delta(S P$, blce & $\mathrm{P}$ \\
$\delta\left(P, r e a^{i}\right)$ & $\mathrm{P}$ & $\delta\left(P, r e f^{i}\right)$ & $\mathrm{SP}$ & $\delta\left(F J, p e r^{i}\right)$ & $\mathrm{SP}$ \\
$\delta\left(P, g o^{i}\right)$ & $\mathrm{G}$ & $\delta\left(S P, f a r^{i}\right)$ & $\mathrm{SP}$ & $\delta\left(F, s d v^{i}\right)$ & $\mathrm{SP}$ \\
$\delta\left(P, f a p^{i}\right)$ & $\mathrm{F}$ & $\delta\left(S P, b l s e^{i}\right)$ & $\mathrm{SP}$ & $\delta\left(F, s d u^{i}\right)$ & $\mathrm{TA}$ \\
$\delta\left(P, f u j^{i}\right)$ & $\mathrm{FJ}$ & $\delta\left(S P, d b^{i}\right)$ & $\mathrm{SP}$ & $\delta\left(F, s d d^{i}\right)$ & $\mathrm{STA}$ \\
$\delta\left(P, d^{i}\right)$ & $\mathrm{SP}$ & $\delta\left(S P, p e c^{i}\right)$ & $\mathrm{SP}$ & $\delta\left(F, s f^{i}\right)$ & $\mathrm{TR}$ \\
$\delta\left(P, p l^{i}\right)$ & $\mathrm{SP}$ & $\delta\left(S P, r e p^{i}\right)$ & $\mathrm{SP}$ & $\delta\left(T A, p e r^{i}\right)$ & $\mathrm{SP}$ \\
$\delta\left(P, p c^{i}\right)$ & $\mathrm{SP}$ & $\delta\left(S P, m a c^{i}\right)$ & $\mathrm{F}$ & $\delta\left(S T A, s a^{i}\right)$ & $\mathrm{TR}$ \\
$\delta\left(P, c e^{i}\right)$ & $\mathrm{SP}$ & $\delta\left(S P, r^{i}\right)$ & $\mathrm{P}$ & $\delta\left(T R, e x p^{i}\right)$ & $\mathrm{X}$ \\
\hline
\end{tabular}

sustenta en una estructura de datos que maneja cada jugada y estado actual del AF. El algoritmo 1 describe la transición y secuencia de jugadas y 2 se muestran los subprocesos necesarios para hacer las transiciones mencionadas. Conviene observar que, sólo utilizando el AF, la ocurrencia de jugadas es correcta y acorde a la reglas del futbol, por ejemplo: una recuperación - un pase - una recepción un pase - ... - un gol.

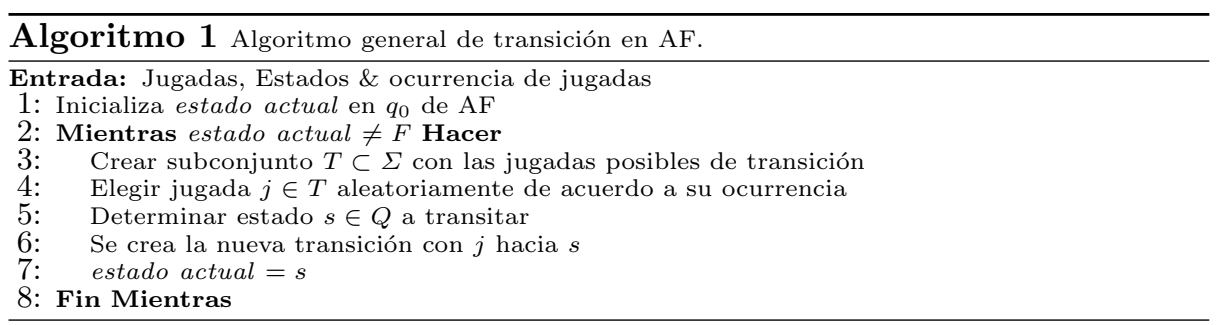

El autómata descrito previamente da secuencias de jugadas para un jugador de FS. Sabemos que la ocurrencia de jugadas, si bien compartidas para delanteros, mediocampistas y defensas, es diferente en frecuencia de ocurrencia para cada rol de juego; el portero tiene un conjunto propio de jugadas. A continuación se muestra una cadena de jugadas para un delantero promedio:

$$
p c^{f} r^{f} p l^{f} \operatorname{far}^{f} \operatorname{far}^{f} r^{f} \operatorname{ref}^{f} \operatorname{far}^{f} b l c e^{f} p c^{f} d b^{f} r^{f} p c^{f} r^{f} p l^{f} r^{f} g o^{f}[
$$

Para esta cadena de jugadas que finalizó en gol se siguieron las reglas de transición:

- $\delta\left(P, p c^{i}\right)=S P$

- $\delta\left(S P, r^{i}\right)=P$

${ }^{1}$ En este ejemplo se utilizó el superíndice $f$ para denotar que se trata del delantero (forward, en inglés). 


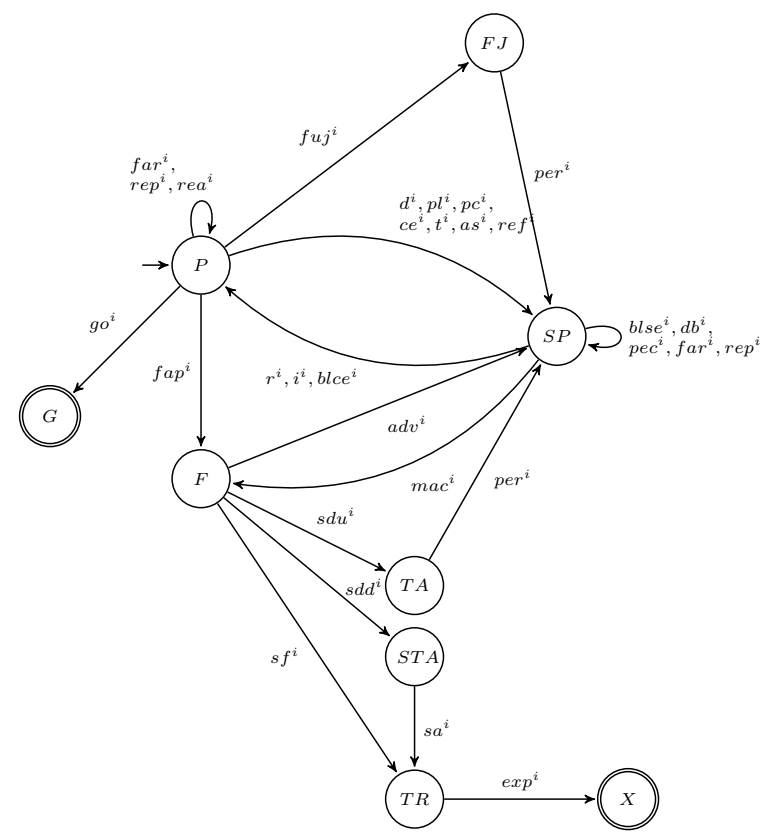

Fig. 1. Autómata Finito para un jugador de fútbol.

- $\delta\left(P, p l^{i}\right)=S P$

- $\delta\left(S P, f^{2} r^{i}\right)=S P$

- $\delta\left(P, r e f^{i}\right)=S P$

- $\delta\left(S P, b l c e^{i}\right)=P$

- $\delta\left(P, g o^{i}\right)=G$

- ...

En contraste con una cadena de jugadas para un defensa promedio :

$p c^{d} r^{d} p c^{d} b l c e^{d} p c^{d} r^{d} p c^{d} r^{d} p c^{d} b l c e^{d} p c^{d}$ far $^{d} b l c e^{d} p c^{d} r^{d} p c^{d} r^{d} p c^{d} b l s e^{d} r^{d} g o^{2}{ }^{2}$

De acuerdo a los datos obtenidos, por ejemplo, un defensa realiza más jugadas de pase corto. Las secuencias de jugadas mostradas previamente se generaron a partir de los algoritmos, y corresponden a cadenas con pocas jugadas. Usualmente, en su mayoría, las cadenas obtenidas tienen más de 100 jugadas.

Debemos observar que las secuencias de jugadas ilustradas antes son sin considerar la frecuencia de ocurrencia real de cada jugada. Es necesario considerar las estadísticas de cada una de las jugadas, y combinar el análisis estadístico previo con las transicisones dadas por el AF.

\footnotetext{
${ }^{2}$ En este ejemplo se utilizó el superindice $d$ para denotar que se trata del defensa(defense, en inglés).
} 
Modelado y análisis formal de jugadas del fútbol

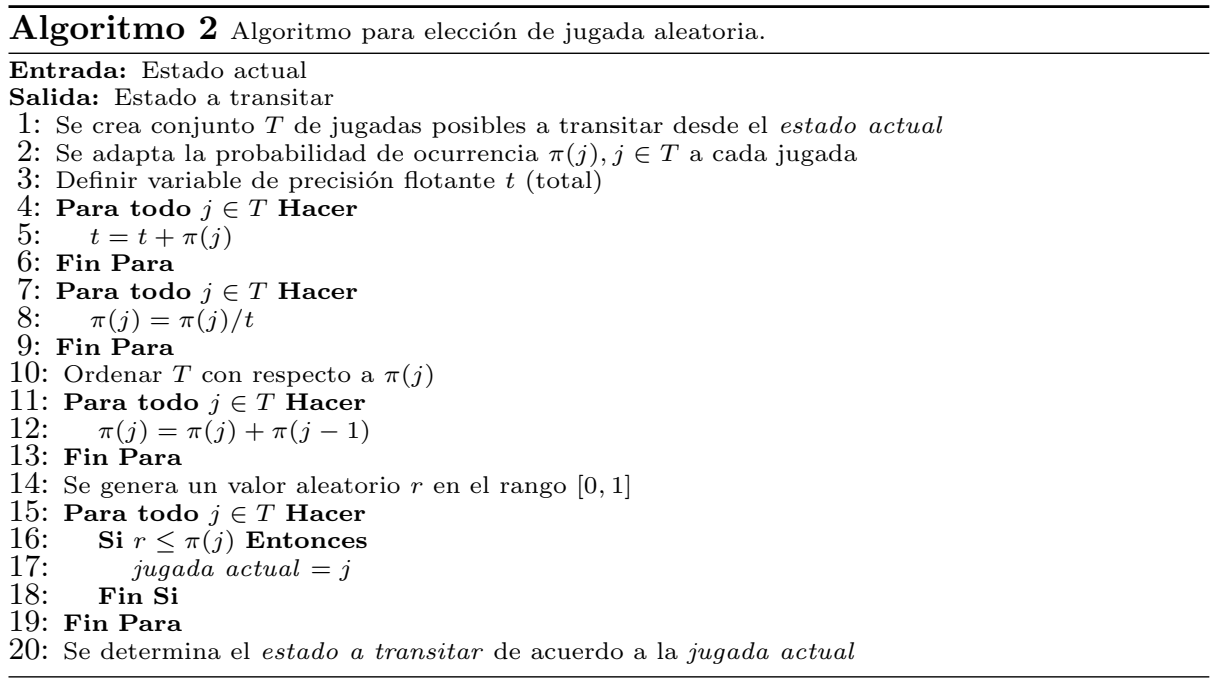

\subsection{El fútbol como sistema concurrente}

Para modelar un partido de FS en su dinámica colectiva se requiere un sistema concurrente. Cada hilo del sistema simula un jugador en el juego. La región critica de concurrencia computacional, se asocia a la posesión del balón. La región critica es compartida por los autómatas - hilos - jugadores, y el acceso a ella implica la posesión del balón, por un único jugador en cada jugada. El control de la región critica en el autómata es de tal forma que mientras un jugador se encuentre en el estado $P$ (Posesión del balón) el resto de los jugadores están en el estado $S P$ (Sin posesión del balón). La transición de un estado a otro es tal que si el jugador $i$ en $P$ hace un pase corto $p c^{i}$, y el jugador $k$ hace una recuperación $r^{k}$, el autómata de cada jugador refleja la acción de él, y entre ambos modelan un movimiento concurrente, de pase corto, como se ilustra en la Figura 2. En la región critica se bloquea la transición si no se cumplan las condiciones para realizar un pase.
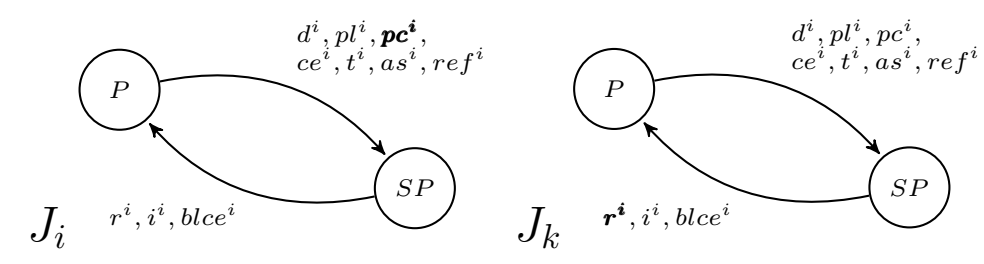

Fig. 2. El sistema concurrente de autómatas de 2 jugadores para un movimiento de pase corto. 
La representación de concurrencia entre las jugadas, con o sin posesión del balón, destaca el modelado simultaneo de jugadas en un partido de futbol, y como cada jugada individual depende de las jugadas de los compañeros en la cancha. Similarmente, una falta propia $\left(f a p^{i}\right)$ la hace un jugador y otro recibe falta $\mathrm{far}^{k}$, y se maneja en hilos concurrentes. Aún en juegos con una dinámica pausada como el béisbol, las jugadas suelen ocurrir concurrentes y en dependencia de las de otro(s) jugador(es): usualmente, un corredor avanza entre las bases 1 - 2 - 3 home una vez que el bateador (pitcher) da un golpe válido a la bola, y éste de manera concurrente, avanza de home a 1, si bien, hay jugadas estrictamente secuenciales, e.g., el golpe a la bola del primer bateador y su avance a primera base, y más si el golpe fue homerun.

\section{Conclusiones}

La habilidad de un jugador en el FS, se caracterizan midiendo la ocurrencia promedio de jugadas por minuto, OPM, que dicho jugador realiza. La dinámica del FS se modela con autómatas concurrentes, un autómata por jugador, y la posesión del balón corresponde a la región critica. Con base en autómatas concurrentes y la OPM la simulación de un partido de FS con jugadores especificos, puede hacerse para equipos reales.

\section{Referencias}

1. Alvarado, M., Rendón, A.Y.: Nash equilibrium for collective strategic reasoning. Expert Syst. Appl. 39(15), 12014-12025 (2012), http://dx.doi.org/10.1016/j. eswa.2012.03.050

2. Alvarado, M., Rendón, A.Y., Cocho, G.: Simulation of baseball gaming by cooperation and non-cooperation strategies. Computación y Sistemas 18(4) (2014), http: //cys.cic.ipn.mx/ojs/index.php/CyS/article/view/1987

3. McDougall, C.: Soccer. Best Sport Ever eBook Series, ABDO Publishing Company (2012), https://books .google.com.mx/books?id=2nrhG5ovUSQC

4. Rendón, A.Y., Rodríguez, R., Alvarado, M.: Analysis of strategies in american football using nash equilibrium. In: Agre, G., Hitzler, P., Krisnadhi, A.A., Kuznetsov, S.O. (eds.) Artificial Intelligence: Methodology, Systems, and Applications - 16th International Conference, AIMSA 2014, Varna, Bulgaria, September 11-13, 2014. Proceedings. Lecture Notes in Computer Science, vol. 8722, pp. 286-294. Springer (2014), http://dx.doi.org/10.1007/978-3-319-10554-3_30 